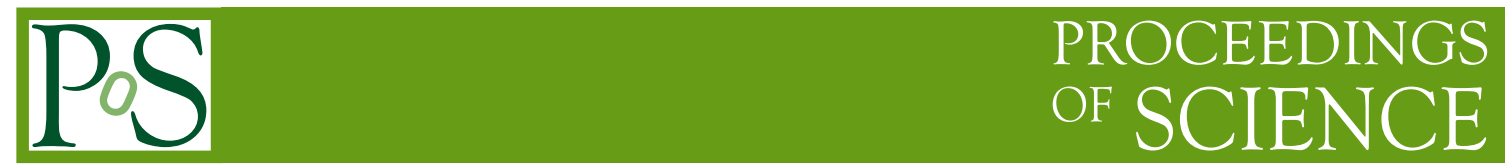

\title{
Measurement of the top quark mass and width with CDF detector
}

\section{Hyun Su Lee* On Behalf of the CDF Collaboration}

Physics Department, Ewha Womans University

E-mail: hyunsuleedewha.ac.kr

\begin{abstract}
The top quark mass is a fundamental parameter of the Standard Model. The precision measurement of the top quark mass combined with the $W$ boson mass measurement can constrain the mass range of the Standard Model Higgs boson. We present updated top quark mass measurements using the full sample of Tevatron $\sqrt{s}=1.96 \mathrm{TeV}$ proton-antiproton collisions collected by the CDF II detector, corresponding to an integrated luminosity of $8.7 \mathrm{fb}^{-1}$.
\end{abstract}

36th International Conference of High Energy Physics,

July 4-11, 2012

Melbourne, Australia

\footnotetext{
* Speaker.
} 


\section{Introduction}

The top quark $(t)$ is by far the heaviest known elementary particle [四]. It contributes significantly to electroweak radiative corrections relating the top quark mass $\left(\mathrm{M}_{\text {top }}\right)$ and the $W$ boson mass to the mass of the Higgs boson []. Precision measurements of $\mathrm{M}_{\text {top }}$ provide therefore an important constraint on the Higgs boson mass. The CDF and D0 experiments have been improving the precision of the $\mathrm{M}_{\text {top }}$ measurement and obtain below $1 \mathrm{GeV} / c^{2}$ precision in the combination of all measurements using data up to $5.6 \mathrm{fb}^{-1}$ [3]. In this report, we present a couple of new $\mathrm{M}_{\text {top }}$ measurements using the full data set of the Tevatron Run II obtained by CDF detector which are not included in the previous Tevatron combination of $\mathrm{M}_{\mathrm{top}}$.

\section{Top quark production and decay}

Top quarks at the Tevatron are predominantly produced in pairs $(t \bar{t})$. Assuming unitarity of the quark-mixing matrix, the top quark decays almost exclusively into a $W$ boson and a $b$ quark. Therefore the topology of $t \bar{t}$ events depends on the different decay of the two $W$ bosons. The lepton+jets channel has one $W$ boson decaying leptonically (an electron or a muon plus a neutrino) and the other hadronically (two quark jets) so that we have one charged lepton, two $b$-quark jets, two light quark jets, and one undetected neutrino. Because of the relatively large branching fraction with manageable backgrounds, we made the most precise $\mathrm{M}_{\text {top }}$ measurements using events in the lepton+jets decay topology.

In the lepton+jets events, some events can not be fully reconstructed because of a missing lepton. They are caused by too small energy deposition in the detector or trajectory outside of detector coverage. There are also hadronically decaying $\tau$ events which we can not reconstruct a charged electron or muon. In these cases, we only reconstruct two $b$-quark jets, two light quark jets, and one undetected $W$ boson. We veto events with a charged lepton to be orthogonal with lepton+jets events. This decay topology is called as $\mathbb{E}_{T}+\mathrm{jets}$ channel.

To improvement the $\mathrm{M}_{\mathrm{top}}$ measurement, CDF collaboration identify $b$ quarks using the properties of the longer lifetime of metastable B hadrons [四]. Therefore jets arising from $b$ quarks have secondary vertices that are displayed from the primary collision vertex. $b$-tagging significantly improve not only background fraction but also the combinatorics of jet-to-parton assignments, improving $\mathrm{M}_{\text {top }}$ resolution.

In the lepton+jets and $\mathbb{E}_{T}$ +jets channels, we have one $W$ boson decaying hadronically ( $W$ decaying to two jets). Therefore we use the reconstructed dijet mass from $W$ boson decay to constrain, in situ, the largest systematic in $\mathrm{M}_{\text {top }}$ measurements, the jet energy scale (JES), which is the calibration between jets energies and parton level energies, because of the narrow decay width and well known mass of the $W$ boson.

\section{Top quark mass measurement}

In the measurements reported in this presentation, we reconstruct top quark masses in each event and compare the distribution of data with template distributions derived from model calculations. This technique is called as template method. We performed $\mathrm{M}_{\mathrm{top}}$ measurements using 

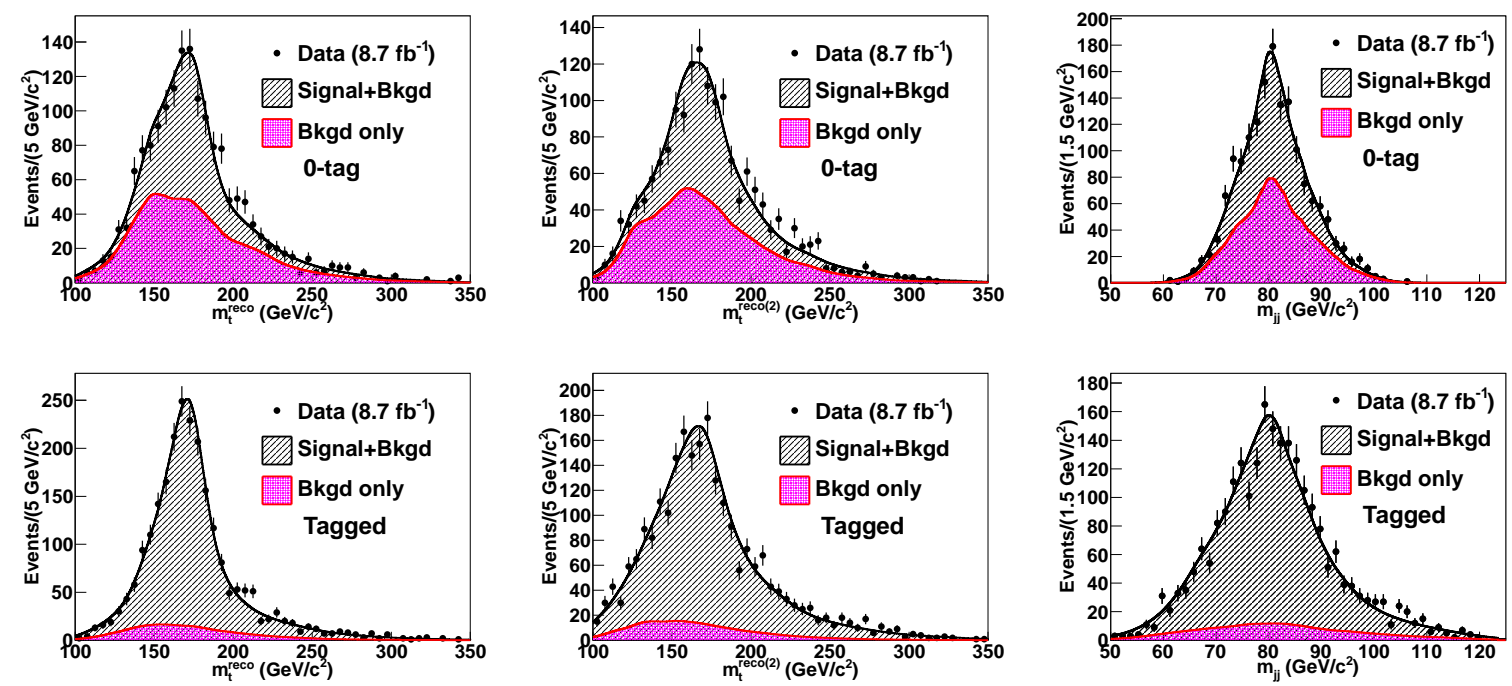

Figure 1: Distributions of the three variables $m_{t}^{\text {reco }}, m_{j j}$, and $m_{t}^{\text {reco(2) }}$, used to measure $\mathrm{M}_{\text {top }}$ for 0-tag and tagged events. The data are overlaid with the predictions from the kernel density estimation probability distributions using $\mathrm{M}_{\mathrm{top}}=173 \mathrm{GeV} / \mathrm{c}^{2}$ and the full background model.

the template method with full data set corresponding to an integrated luminosity of $8.7 \mathrm{fb}^{-1} p \bar{p}$ collisions at CDF.

In the lepton+jets channel, we reconstruct top quark masses $\left(m_{t}^{\text {reco }}\right.$ and $\left.m_{t}^{\text {reco(2) }}\right)$ using kinematic fitter [[]]. We also use the template distributions of hadronically decaying $W$ bosons $\left(m_{j j}\right)$ to constrain the jet energy scale, an important uncertainty in the $\mathrm{M}_{\text {top }}$ measurement. This is an update of a previous measurement that used a subset of the present data [6]. In this measurement, we use not only larger samples but also improve jet energy calibration using an artificial neural network $[\nabla]$ to achieve better jet energy resolution, and increase signal acceptance allowing less pure signal samples into the analysis. With the maximum likelihood fit to the data with signal and background templates, we measure

$$
\mathrm{M}_{\mathrm{top}}=172.85 \pm 0.71 \text { (stat) } \pm 0.85 \text { (syst) } \mathrm{GeV} / c^{2}=172.85 \pm 1.11 \mathrm{GeV} / c^{2}
$$

This is the most precise $\mathrm{M}_{\text {top }}$ measurement in the single channel to date [ [ $]$ ]. Figure $\square$ shows the distributions of the observables used for the $\mathrm{M}_{\mathrm{top}}$ measurement in the lepton+jets channel, overlaid with density estimates using $t \bar{t}$ signal events with $\mathrm{M}_{\mathrm{top}}=173 \mathrm{GeV} / \mathrm{c}^{2}$ (close to the measured $\mathrm{M}_{\mathrm{top}}$ ) and the full background model.

In the $\mathbb{E}_{T}+$ jets channel, we can not detect a lepton and a neutrino from single $W$ boson. Because of two missing particles in the leptonic side, we used the reconstructed masses of the hadronically decaying top quark from three jets making the largest transverse momentum, $p_{T}$, in the previous measurement [Q]. However, we still have measured information not used in the reconstruction of the hadronically decaying top quark. For the top quark mass information, the reconstruction of a lepton and a neutrino is not necessary. By assuming of a single missing particle, leptonically decaying $W$ boson, we reconstruct $t \bar{t}$ events using $\chi^{2}$-like kinematic fitter and form 

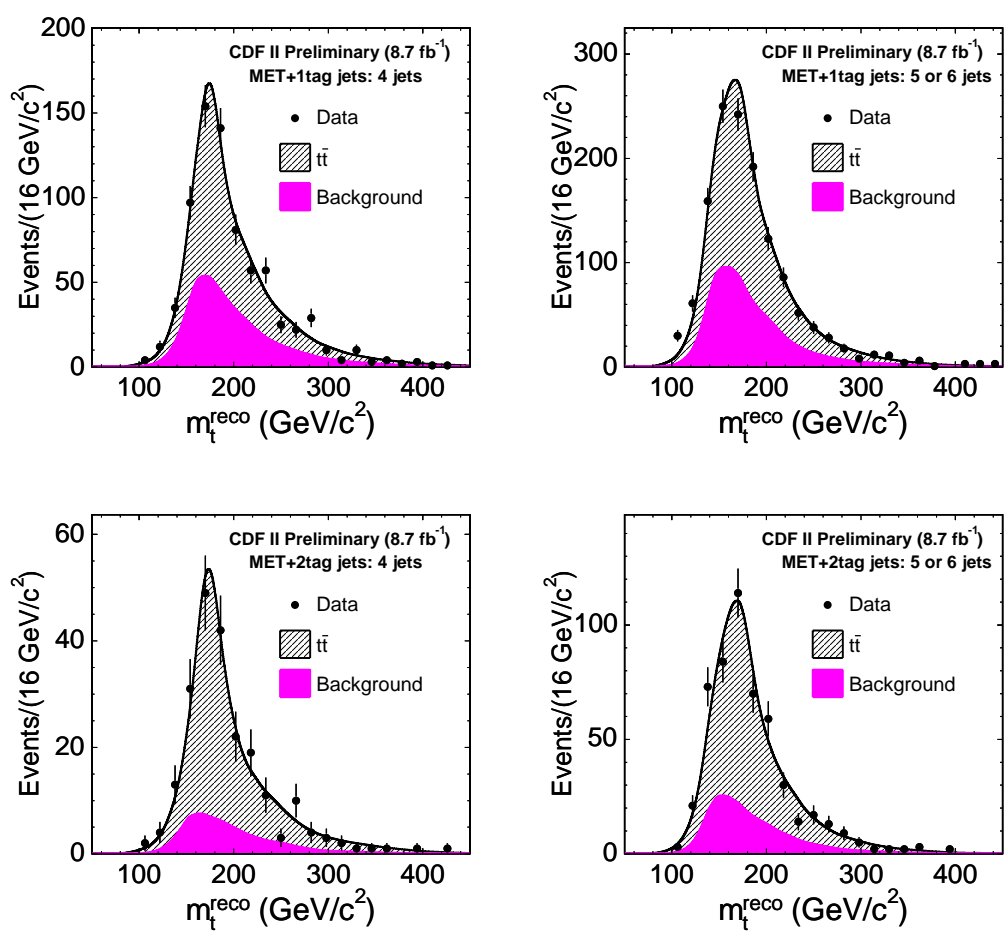

Figure 2: Distributions of the reconstructed top quark mass, used to measure $\mathrm{M}_{\mathrm{top}}$ in the $\mathbb{E}_{T}+$ jets channel for 1-tag and 2-tag events (4 jet and 5,6 jet evnets separately). The data are overlaid with the predictions from the kernel density estimation probability distributions using $\mathrm{M}_{\mathrm{top}}=173.5 \mathrm{GeV} / \mathrm{c}^{2}$ and the full background model.

the estimators of the top quark mass. We use three variables, two of the reconstructed top quark masses $\left(m_{t}^{\text {reco }}\right.$ and $\left.m_{t}^{\text {reco(2) }}\right)$ and hadronically decaying $W$ boson mass $\left(m_{j j}\right)$ to estimate $\mathbf{M}_{\text {top }}$ and constrain the jet energy scale. Applying a maximum likelihood fit to data, we measure [ய]]

$$
\mathrm{M}_{\text {top }}=173.9 \pm 1.6 \text { (stat) } \pm 0.9 \text { (syst) } \mathrm{GeV} / c^{2}=173.9 \pm 1.9 \mathrm{GeV} / c^{2}
$$

Figure $\square$ shows the distributions of the reconstructed top quark mass used for the $\mathrm{M}_{\text {top }}$ measurement in the $\mathbb{E}_{T}+$ jets channel, overlaid with density estimates using $t \bar{t}$ signal events with $\mathrm{M}_{\text {top }}=$ 173.5 GeV $/ c^{2}$ (close to the measured $\mathrm{M}_{\mathrm{top}}$ ) and the full background model.

The two of new measurements performed with the full data set of CDF Run II improve the precision of $\mathrm{M}_{\mathrm{top}}$ and are in agreement of the Tevatron average [B]].

\subsection{Top and anti-top quark mass difference measurement}

The precision determination of $\mathrm{M}_{\text {top }}$ allows us to measure the mass difference between top quark and anti-top quark to a similar precision. In the CPT theorem, which is fundamental to any local Lorentz-invariant quantum field theory, the particle mass should be same as its anti-particle partner. Despite the fact that no violations have ever been observed in the meson and baryon sectors, it is important to test CPT violation in all sectors such as quarks and high mass particles. 

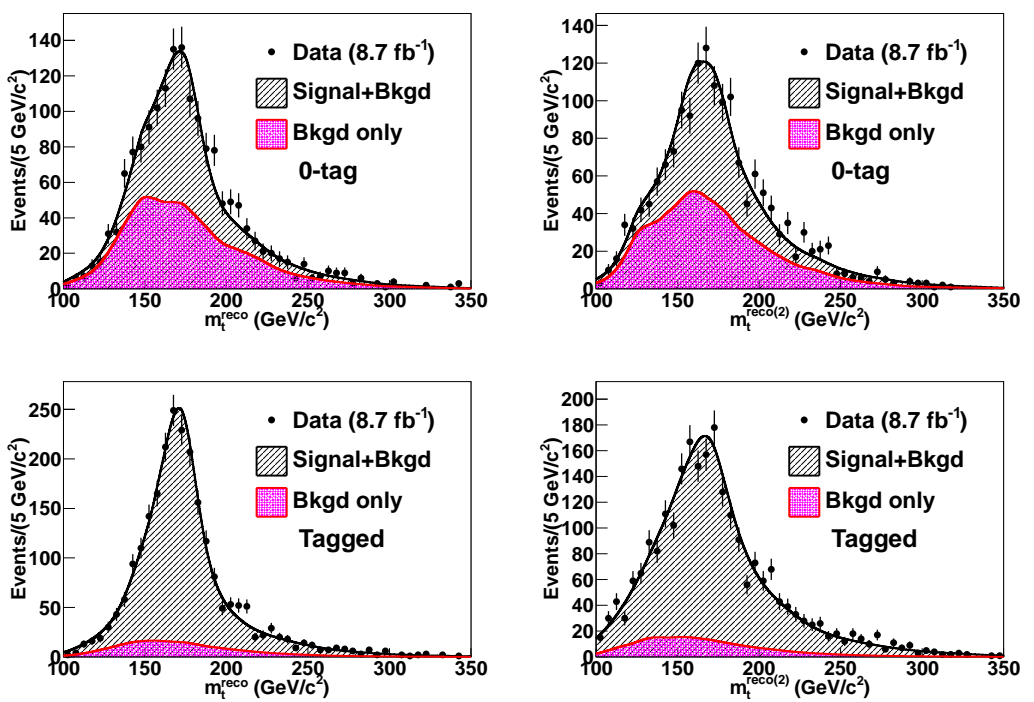

Figure 3: Distributions of two reconstructed mass difference $\left(\Delta m_{t}^{\text {reco }}\right.$ and $\left.\Delta m_{t}^{\text {reco(2) }}\right)$ used to extract the mass difference between $t$ and $\bar{t}\left(\delta \mathrm{M}_{\mathrm{top}}\right)$ for zero $b$-tagged ( 0 -tag) events and one or more $b$-tagged (Tagged) events. The data are overlaid with predictions from the kernel density estimation probability distributions assuming $\delta \mathrm{M}_{\mathrm{top}}=0 \mathrm{GeV} / c^{2}$. The fitted number of signal and background events are used.

CDF collaboration measure the mass difference between top quark and anti-top quark $\left(\delta \mathrm{M}_{\mathrm{top}}\right.$ ) in the lepton+jets channel using the template technique. We reconstruct the mass difference $\left(\Delta m_{t}^{\text {reco }}\right.$ and $\Delta m_{t}^{\text {reco(2) }}$ ) using modified kinematic fitter allowing mass difference between hadronic top quark and leptonic top quark. Using $5.6 \mathrm{fb}^{-1}$ of $p \bar{p}$ collisions, we measured $\delta \mathrm{M}_{\mathrm{top}}=-3.3 \pm$ $1.7 \mathrm{GeV} / c^{2}[\mathbb{[}]$. In the update measurement using the full data set, we apply the two improvements introduced for the $\mathrm{M}_{\text {top }}$ measurement. First, we employ the neural network jet energy scale correction and improve the resolution of the jet. Second, we increase the signal acceptance allowing less pure signal samples. The resulting mass difference from $8.7 \mathrm{fb}^{-1}$ data [미] is

$$
\delta \mathrm{M}_{\text {top }}=-1.95 \pm 1.11 \text { (stat) } \pm 0.59 \text { (syst) } \mathrm{GeV} / c^{2}=-1.95 \pm 1.26 \mathrm{GeV} / c^{2} .
$$

Figure [3] shows the observed distributions of the observables used for the $\delta \mathrm{M}_{\text {top }}$ measurement. The density estimates for $t \bar{t}$ signal events with $\delta \mathrm{M}_{\mathrm{top}}=0 \mathrm{GeV} / c^{2}$ and for background events are overlaid. This result is consistent with $\delta \mathrm{M}_{\mathrm{top}}=0 \mathrm{GeV} / c^{2}$ and conservation of CPT symmetry.

\section{Conclusion}

The CDF collaboration has updated a robust set of $\mathrm{M}_{\text {top }}$ and $\delta \mathrm{M}_{\text {top }}$ measurements with full CDF Run II data set with significant improvements.

\section{Acknowledgments}

I would like to thank for the CDF colleagues for their efforts to carry out these challenging physics analyses. My travel was supported by the National Research Foundation of Korea Grant funded by the Korean Government [NRF-2011-35B-C00007]. 


\section{References}

[1] K. Nakamura et. al. (Particle Data Group), J. Phys. G 37, 075021 (2010).

[2] ALEPH, CDF, D0, DELPHI, L3, OPAL, SLD, the LEP Electroweak Working Group, the Tevatron Electroweak Working Group, and the SLD Electroweak and Heavy Flavor Working Groups, arXiv:1012.2367v2.

[3] T. Aaltonen et. al. (CDF and D0 Collaborations), Phys. Rev. D 86, 092003 (2012).

[4] D. Acosta et. al. (CDF Collaboration), Phys. Rev. D 71, 052003 (2005).

[5] A. Abulencia el. al. (CDF Collaboration), Phys. Rev. D 73, 032003 (2006).

[6] T. Aaltonen et. al. (CDF Collaboration), Phys. Rev. D 83, 111101 (2011).

[7] T. Aaltonen, A. Buzatu, B. Kilminster, Y. Nagai, and W. Yao, arXiv:1107.3026.

[8] T. Aaltonen et. al. (CDF Collaboration), Phys. Rev. Lett. 109, 152003 (2012).

[9] T. Aaltonen et. al. (CDF Collaboration), Phys. Rev. Lett. 107, 232002 (2011).

[10] CDF Collaboration, CDF Publicnote 10810.

[11] T. Aaltonen et. al. (CDF Collaboration), Phys. Rev. Lett. 106, 152001 (2011).

[12] T. Aaltonen et. al. (CDF Collaboration), arXiv:1210.6131. 